\title{
Rugoscopy: A Diagnostic Appurtenance for Malocclusion or just a Forensic Aid? - A Pilot Study
}

Priyanka Kapoor*, Ragini and Harneet Kaur

Department of Orthodontics, Faculty of Dentistry, Jamia Millia Islamia, New Delhi, India

\begin{abstract}
Background: Palatal rugae (PR) routinely used for forensic identification of individuals, have limited use in orthodontics as a stable reference for cast superimpositions. PR shape gets established early in intra-uterine life and remains stable thereafter, thus its correlation with different occlusal relations may aid in early interception and prevention of malocclusions.
\end{abstract}

Aims: Identification of characteristic features of PR in different classes of malocclusion (Angle's Class I, II and III) and to explore its potential as an adjunctive aid for categorizing malocclusions.

Objective: To explore the potential of PR as an adjunctive aid for categorizing malocclusions.

Materials and methods: PR in pre-treatment study casts of 66 patients (age 12-26 years) demonstrating Class I (type1 and type2), Class II div1, Class II div2 and Class III malocclusions were traced. The number, pattern, orientation and strength of rugae both on left and right side of mid-palatal raphe were determined and statistically analysed with non-parametric Kruskal Wallis test for different categories of malocclusions.

Results: Class II div2 patients showed largest number of primary, secondary and fragmentary rugae, although it was not statistically significant. PR in all malocclusions showed a predominantly curvy pattern but in Class I and Class III patients on left side, forking-diverging type was mainly seen. Horizontal orientation of PR was characteristically absent in Class II div1 patients. Strength of rugae became strong to weak from first to third primary rugae.

Conclusion: This pilot study provides evidence of a distinct pattern of PR in Class I and Class III patients and largest number in Class II div2 cases but to ascertain its statistical significance, further research may be conducted with a larger sample size.

Keywords: Palatal rugae; Malocclusion; Classification, Forensic identification; Adjunct

\section{Introduction}

Transverse palatine folds or palatal rugae (PR), are asymmetrical and irregular elevations of the mucosa in the anterior third of the palate arranged in a transverse direction on each side of the medial palatal raphae and behind the incisive papilla (IP), located in the mid-sagittal plane [1,2]. Developmentally, palatal rugae are formed in the third month in utero from the hard connective tissue covering bone. First $\mathrm{PR}$ were identified in human embryos of 32-mm Crown-rump length (CRL) next to IP [3]. The orientation pattern is formed by about $12^{\text {th }}$ to $14^{\text {th }}$ week of prenatal life and remains stable until the oral mucosa degenerates after death $[4,5]$. The prominence of PR diminishes from prenatal period to adolescence but the shape remains unaltered [6].

The post mortem resistance, stability and uniqueness of palatal rugae make them a potential adjunct for forensic identification. Ample evidence in literature supports stability in shape of PR, although increase in size of anterior part of the palate in early years of life may bring about change in length of PR $[7,8]$. Gender differentiation is also noticed in some longitudinal studies for increase in length of PR from 5 to 10 years of age, notably $11 \%$ for boys and $9 \%$ for girls [5,9]. A more or less continuous and small increase in distances between medial borders of paired rugae have also been reported along with a backward extension of posterior boundary of PR in relation to teeth until 20 years of age [10].

Although, some habits like finger sucking or persistent pressure from orthodontic treatment or dentures and orthodontic extractions may bring about local changes in PR [2,11], the stability in shape of PR has been routinely exploited for dental cast superimposition to monitor tooth movement in orthodontics [12-14]. Besides, an appreciable hereditary component of palatal rugae has also been suggested in various twin and family studies [15-17] but this varies in extent and characteristics in different investigations.

The stability and hereditary inclination exhibited by PR led to formulation of hypothesis that various occlusal characteristics that display a strong genetic basis may display a correlation with PR. This in turn may serve as a diagnostic appurtenance for malocclusions very early in life and aid in preventive or interceptive measures to alleviate forthcoming dento-skeletal aberrations.

\section{Material and Methods}

The present study was conducted after obtaining study records of 66 young adult patients (12-26 years) who reported to the OPD of Department of Orthodontics following institutional ethical clearance. The inclusion criteria for the sample were: complete complement of

*Corresponding author: Dr. Priyanka Kapoor MDS (Orthodontics, AlIMS), Associate Professor, Department of Orthodontics, Faculty of Dentistry, Jamia Millia Islamia, New Delhi-110025, India, Tel: 98191914997; E-mail: pkapoor@jmi.ac.in

Received January 15, 2015; Accepted February 19, 2015; Published February 25, 2015

Citation: Kapoor P, Ragini, Kaur H (2015) Rugoscopy: A Diagnostic Appurtenance for Malocclusion or just a Forensic Aid? - A Pilot Study. J Forensic Res 6: 272. doi: 10.4172/2157-7145.1000272

Copyright: ( 2015 Kapoor $P$, et al. This is an open-access article distributed under the terms of the Creative Commons Attribution License, which permits unrestricted use, distribution, and reproduction in any medium, provided the original author and source are credited. 
permanent teeth till second molars; no history of previous extraction of teeth/orthodontic treatment; no deleterious habits and no congenital/ acquired deformity of head and neck. Irrespective of gender, the sample was classified into 4 groups based on Angle's classification of malocclusion: Class I comprising of Class I type1 and type2 $(\mathrm{N}=24)$; Class II div1 ( $\mathrm{N}=20)$; Class II div $2(\mathrm{~N}=10)$; Class III $(\mathrm{N}=12)$.

All study casts were poured with type III dental stone and labelled according to name, age, sex and type of malocclusion. Landmarks on palatal raphe and PR were marked using a $0.3 \mathrm{~mm}$ graphite pencil according to the classification given by Kapali et al. [18] (Figure 1). All markings were then traced on to a transparent template (Figure 2). The traced palatal rugae were observed for length, orientation/direction, shape/pattern and strength/thickness as follows:

\section{Length of PR (Classification)}

PR were classified according to length, as given by Lysell in 1955 [2]. It was recorded using digital vernier calipers (least count $=0.05$ $\mathrm{mm}$ ) from medial end of rugae to the lateral end.

1. Primary rugae [2]/Main ridges [19]: (5mm or more)

2. Secondary rugae [2]/Secondary ridges [19]: 3-5 mm

3. Fragmentary rugae [2]/ridges: less than $3 \mathrm{~mm}$.

The number of rugae falling under the category of primary, secondary and fragmentary rugae was recorded separately for left and right side of each study cast.

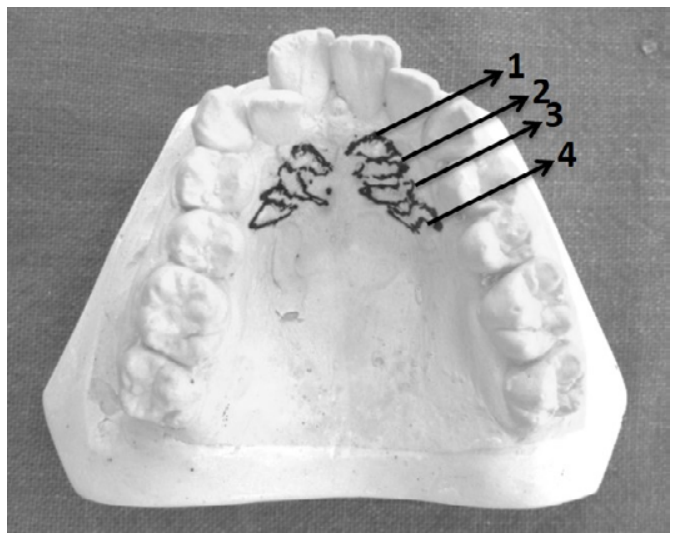

Figure 1: Primary, secondary and fragmentary rugae traced on each side of mid palatal raphe (numbering starting anteriorly, next to the incisive papilla).

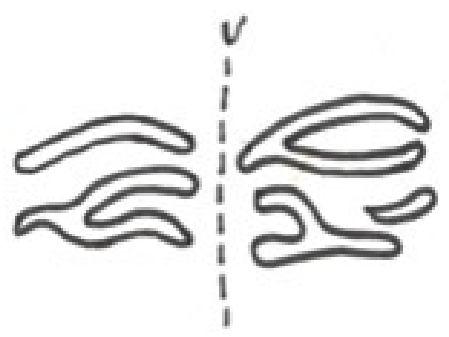

Figure 2: Transparent template made to study orientation, shape and strength of palatal rugae.

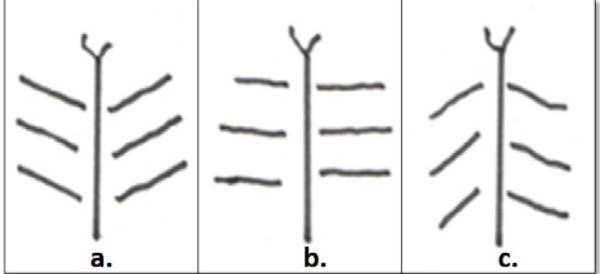

Figure 3: Orientation (direction) of first, second and third Primary rugae of right and left side of mid-palatal raphe recorded separately: a. Posteriorlyb. Horizontal c. Anteriorly.

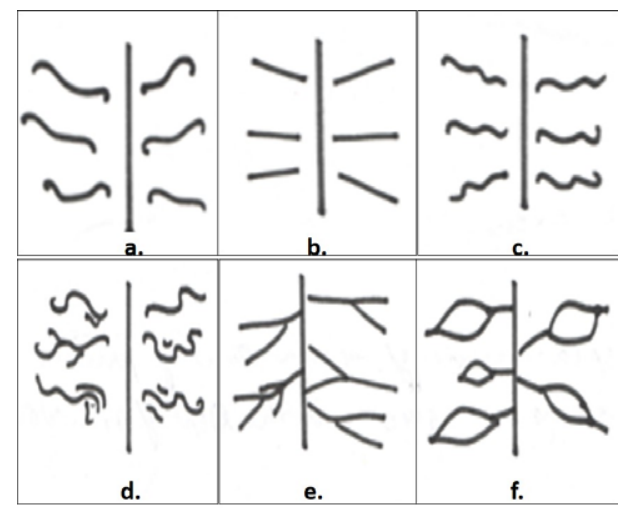

Figure 4: Pattern/ Shape of first, second and third Primary rugae of right and left side of mid-palatal raphe recorded separately: a. Curved(c) b. Straight(s) c. Wavy (w) d. Irregular (Ir)e. Forking (f)f. Island (i)

\section{Orientation or direction of palatal rugae}

The orientation/direction of first, second and third primary rugae were recorded separately for left and right side of each cast where an angle was formed between mid-palatal raphe and PR. It was classified according to Hauser as follows [19]: (Figure 3)

1. Posteriorly directed rugae $(\mathrm{p})$ - associated with negative angles (Figure 3a).

2. Horizontal/perpendicular rugae $(\mathrm{h})$ - associated with angles of zero degrees (Figure $3 \mathrm{~b}$ ).

3. Anteriorly directed rugae (a) - associated with positive angle (Figure 3c).

\section{Shape/Pattern of palatal rugae}

Using the template tracings, shape of first, second and third primary rugae were recorded separately for left and right side of each cast from their origin to termination and classified according to Hauser et al. [19] as follows (Figure 4):

1. Curved(c): The curved type had a simple crescent shape which curved gently.

2. Straight(s): Straight types ran straight directly from their origin to termination.

3. Wavy (w): The basic shape of the wavy rugae was serpentine; however, if there was a slight curve at the origin or termination of curved rugae it was classified as wavy. 
4. Forking (f); Unification occurs when two rugae are joined at their origin or termination based on which, they were of two types (Figure 5):

fd1: Converging: Rugae with different origins which joined on their lateral portions.

fd2: Diverging: Unifications in which two rugae began from the same origin but immediately diverged.

1. Island (i): The rugae that displayed a definite continuous ring formation at the termination was termed as Island.

2. Irregular (Ir): Broken, irregular pattern of rugae.

\section{Strength of palatal rugae}

Based on thickness of first, second and third primary rugae strength of were classified according to Hauser et al. [19] as follows (Figure 6):
a. Strong(s)
b. $\operatorname{Medium}(\mathrm{m})$
c. Weak $(w)$

\section{Statistical Analysis}

All data was collected and visually screened for any missing data or outliers. Statistics were performed in SPSS 17.0 (SPSS, Chicago III). Descriptive statistics (mean and standard deviation) were determined

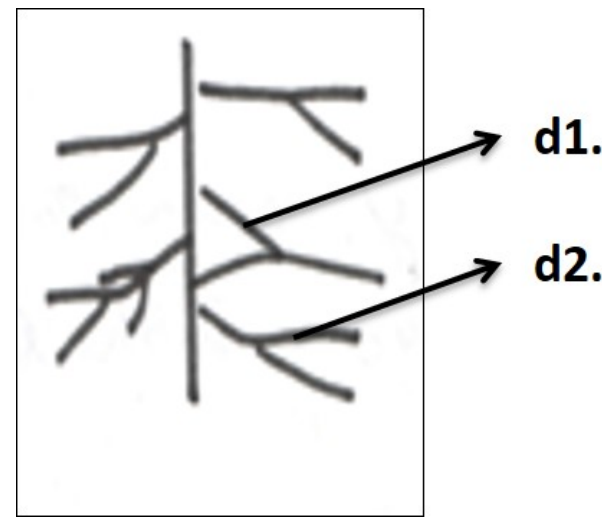

Figure 5: Pattern of unification based on the origin and termination, rugae classified into two types:d1: Converging-type (fd1)d2: Diverging -type (fd2).

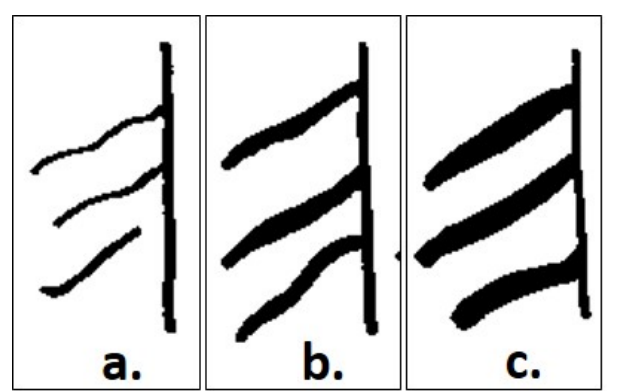

Figure 6: Strength of first, second and third Primary rugae of right and left side of mid-palatal raphe recorded separately: recorded:a. Strong(s)b. Medium(m) c. Weak(w). for the number of palatal rugae (primary, secondary and fragmentary) on each side of mid-palatal raphe for each malocclusion group. The most predominant pattern, orientation and strength of primary rugae (first, second and third) in each malocclusion group was studied. Non parametric test, Kruskal-Wallis was done to find association of independent variables of $P R$ number, pattern, orientation and strength with the various malocclusion groups. $\mathrm{P}$ value $<0.05$ was considered to be statistically significant.

\section{Results}

Results of Table 1 showed number of primary rugae in Class I malocclusion on left side being greater in number with mean $3.13 \pm 0.75$ as compared to right side having mean value of $3 \pm 0.6$. In Class II div 1 and Class III malocclusion, number of primary rugaes were present more left side with values of $3.08 \pm 0.7$ and $3.21 \pm 0.58$ respectively. The mean values of secondary and fragmentary rugae were less than 1 in all the three categories of malocclusion except Class II div 2. The average number of rugae taking into account primary, secondary and fragmentary rugaeis greatest in Class II div2 but almost similar in rest of the classifications. ANOVA did not depict any statistically significant difference in $\mathrm{p}$-vlaue for rugae number.

The results of Table 2 did not depict any particular pattern, orientation or strength for a particular malocclusion. However, the most predominant pattern for first, second and third primary rugae was curved with forking-diverging, being most common in first primary rugae of left side in Class I and Class III malocclusions. The trend of orientation pattern in Class I from first to third rugae predominantly shifts from horizontal to posteriorly while Class II div 1 did not show horizontal pattern in any of the rugae, orientation of rugae in Class II div 2 remained more or less horizontal while in Class III horizontal and posteriorly were two most predominant types. The strength of rugae showed a similar trend for all malocclusions, with the first primary rugae being strong gradually becoming weak till the third rugae.

The results of Table 3 did not depict any significant difference in rugae pattern, orientation and strength among the four malocclusion groups based on the test scores.

\section{Discussion}

Palatal ridges or rugae palatinae are transversal ridges found on the hard palate of most mammalian species, but display exclusivity in number and arrangement for different species [20]. In the present study the average number of primary rugae has been approximately three in number in accordance with human studies done previously [21]. The number of primary rugae was seen to be greatest in Class II div 2 patients, probably due to difference in degree of development of maxillary basal arch width and transverse morphology. Hauser et al. in 1989 that stated that size of the palate affected rugae development with broader palates showing greater rugae development [19]. Studies have compared maxillary arch width development in normal occlusion, Class II div1 and Class II div2 patients and found it in the order of normal occlusion having greatest, followed by Class II div2 and least in Class II div1 patients [22]. This corresponds to our findings wherein normal occlusion sample has not been taken, and amongst the remaining malocclusion, Class II div 2 shows maximum number of rugae. Literature provides evidence of lesser maxillary intercanine and interpremolar arch width development as compared to normal occlusion in Class III samples that supports our finding [23]. Nevertheless, to attain a conclusive evidence of this particular outcome, a larger sample size of Class II div2 has to be studied and compared to normal occlusion patients having similar palatal widths. 
Page 4 of 5

\begin{tabular}{|c|c|c|c|c|c|c|c|c|}
\hline \multirow[t]{2}{*}{ Malocclusion } & \multirow[t]{2}{*}{$\mathbf{N}$} & \multicolumn{2}{|c|}{ No. of primary Rugae } & \multicolumn{2}{|c|}{ No of secondary Rugae } & \multicolumn{2}{|c|}{ No. of fragmentary Rugae } & \multirow[t]{2}{*}{ Mean No. of Rugae } \\
\hline & & Right & Left & Right & Left & Right & Left & \\
\hline Class I & 24 & $3.00 \pm .60$ & $3.13 \pm .75$ & $.65 \pm 1.11$ & $0.26 \pm .62$ & $0.60 \pm 0.83$ & $0.65 \pm 1.027$ & $8.30 \pm 3.11$ \\
\hline Class II div 1 & 20 & $3.00 \pm 0.59$ & $3.08 \pm 0.70$ & $0.44 \pm 0.65$ & $0.36 \pm 0.63$ & $0.56 \pm 0.86$ & $0.60 \pm 0.81$ & $8.04 \pm 2.30$ \\
\hline Class II div 2 & 10 & $4.00 \pm 0.00$ & $4.00 \pm 0.00$ & $1.00 \pm 0.000$ & $1.00 \pm 0.00$ & $2.00 \pm 0.00$ & $2.00 \pm 0.00$ & $14.00 \pm 0.00$ \\
\hline Class III & 12 & $3.00 \pm 0.68$ & $3.21 \pm 0.58$ & $.64 \pm 0.74$ & $0.64 \pm 0.50$ & $0.43 \pm 0.76$ & $0.42 \pm 0.64$ & $8.35 \pm 2.69$ \\
\hline $\mathbf{F}$ & & 1.761 & 1.133 & 0.456 & 1.895 & 2.129 & 1.950 & \\
\hline Sig (p Value) & & 0.164 & 0.343 & 0.714 & 0.140 & 0.106 & 0.131 & \\
\hline
\end{tabular}

Table 1: Mean and standard deviation for number of palatal rugae on right and left side in various malocclusions along with statistical ANOVA test for significance.

\begin{tabular}{|c|c|c|c|c|c|c|c|}
\hline \multirow[t]{2}{*}{ Malocclusion } & \multirow[t]{2}{*}{ Parameter } & \multicolumn{2}{|c|}{$1^{\text {st }}$ Primary Rugae } & \multicolumn{2}{|c|}{$2^{\text {nd }}$ Primary Rugae } & \multicolumn{2}{|c|}{$3^{\text {rd }}$ Primary Rugae } \\
\hline & & Right & Left & Right & Left & Right & Left \\
\hline \multirow[t]{3}{*}{ Class I } & Pattern & (c) & $(\mathrm{fd} 2)$ & (c) & (c) & (c) & (c) \\
\hline & Orientation & (h) & (h) & (a) & $(p)$ & (a) & (p) \\
\hline & Strength & (s) & (s) & $(\mathrm{m})$ & $(\mathrm{m})$ & $(w)$ & (w) \\
\hline \multirow[t]{3}{*}{ Class II div 1} & Pattern & (c) & (c) & (c) & (c) & (c) & (c) \\
\hline & Orientation & (p) & (p) & (a) & $(p)$ & (a) & (a) \\
\hline & Strength & (s) & (s) & (m) & $(\mathrm{m})$ & (m) & (m) \\
\hline \multirow[t]{3}{*}{ Class II div 2} & Pattern & (c) & (c) & (c) & (c) & (c) & (c) \\
\hline & Orientation & (h) & (p) & (h) & (h) & (h) & (h) \\
\hline & Strength & (s) & (s) & (m) & $(\mathrm{m})$ & $(\mathrm{m})$ & (m) \\
\hline \multirow[t]{3}{*}{ Class III } & Pattern & (c) & (fd1) & (c) & (c) & (c) & (c) \\
\hline & Orientation & (h) & (p) & (a) & $(p)$ & (h) & (h) \\
\hline & Strength & (s) & (s) & $(\mathrm{m})$ & $(\mathrm{m})$ & $(w)$ & (w) \\
\hline
\end{tabular}

Table 2: Most common pattern, orientation and strength of primary palatal rugae in various malocclusion groups. Pattern: (c): Curved; (s): straight; (w): wavy; (fd1): forkingconverging; (fd2): forking- diverging; (i): island; (Ir): irregular Orientation: (h): horizontal; (a): anteriorly; (p): Posteriorly Strength; (s): strong; (m): medium; (w): weak.

The age group of 12-26 years was selected based on the evidence that orientation pattern gets established at birth finally attaining a final feature during adolescence and thereafter any change that occurs may be in length but not in shape [7,8]. Thus the shape of PR once ascertained, could be investigated for any correlations with ethnicity or malocclusions. There is ample substantiation of association between PR patterns and number with ethnicity. Kapali et al. [18] compared the rugae pattern in Australian Aborigines and Whites and found that the former displayed more number of primary rugae than the Whites, with wavy and curved forms being more prominent. Similar studies comparing Indian population with Japanese and Tibetan population have also revealed significant differences in PR pattern [24,25]. Thus, prevalent malocclusions in various ethnic populations [26] could also be investigated for a positive correlation with specific pattern, length and number of PR and serve as an adjunct in intercepting malocclusions at a very early age. A single study in literature by Gandikota et al. in 2012 found a distinct palatal rugae pattern in class II div 1 when compared to class I individuals. They also they reported a significant constriction of PR in Class II div1 when evaluated with Class I individuals having same intermolar widths [6]. Their findings did not correspond with the results of our study as they had formulated an ordinal scale to compare the rugae pattern as good to bad while we had compared individual characteristics of pattern, orientation and strength and found no significant correlation in any malocclusion. Also they had compared length of first to third rugae while we had observed number of primary in different categories of malocclusion and found the number to be greatest in Class II div2 patients.

The physiological function of PR in oral swallowing, speech and suction has been well documented [3]. The association of food and taste receptors in the dorsal surface of tongue has been considered responsible for particular configurations and prominence of rugae [3]. And as tongue posture is variant in Class II and Class III patients, it was expected to influence the PR pattern in different categories of malocclusion [27]. In Class II malocclusion groups, contact of anterior portion of the tongue with rugae area of hard palate has been shown to be decreased as compared to the control group [28]. While in Class III malocclusion, tongue-to-palate distances were found to be generally greater and tongue posture lower as compared to controls [27]. Although our study has shown differences in pattern in Class I and Class III casts displaying a forking-diverging type on left side, but it was statistically not significant. Thus future studies should be done with more sample size in consideration.

PR number, shape as well as orientation have shown genetic predisposition in previous studies [15-17], thus supports its correlation with different malocclusions having strong genetic potential. Both Class III and Class II display specific genes leading to a particular skeletal variability caused by polygenic nature of craniofacial traits $[29,30]$ and these could be explored in forthcoming studies for specific association with PR shape and number.

The strength of rugae decreases from first to third primary rugae in our study. This is in accordance with literature evidence of decreasing regularity of rugae pattern, disappearance of posterior rugae and strength of anterior rugae becoming considerably more pronounced and compressed, towards the end of intrauterine life [31].

This pilot study has broadened the scope for future studies after addressing the limitations of this study. First and foremost, a larger sample size is required to authenticate the observations of this study to attain results that are statistically significant. Another important consideration should be equal distribution of male and female samples as studies have proved the uniqueness and gender differentiation of PR pattern in individuals $[32,33]$. Future studies should have a study design comprising of a control sample of untreated normal Class I occlusion patients for validation of association of palatal growth with rugae in malocclusion patients. 


\begin{tabular}{|l|c|c|c|c|c|}
\hline Parameter & \multicolumn{2}{|c|}{ 1 $^{\text {st }}$ Primary Rugae } & \multicolumn{2}{|c|}{ 2 $^{\text {nd }}$ Primary Rugae } & Left \\
\hline Pattern(P) & Right & Left & Right & Right & 0.705 \\
\hline Orientation(Or) & 1.00 & 0.222 & 0.875 & 0.370 \\
\hline Strength(St) & 0.488 & 0.276 & 0.819 & 0.592 & 0.660 \\
\hline
\end{tabular}

Table 3: Kruskal Wallis Test Statistics for association for Rugae Pattern (P), Orientation (Or) and Strength (St) among the four malocclusion groups.

\section{Conclusions}

1. The number of primary, secondary and fragmentary rugae was similar in almost all malocclusions except Class II div 2 that showed the greatest number of all forms of rugae.

2. The pattern of PR has shown distinction in Class III malocclusion (forking diverging type)

3. No statistically significant difference was observed in number, pattern, orientation and strength of first, second and third primary rugae in any of the malocclusion groups.

A major limitation of the study was a small sample size but if the same study is performed on a larger sample size, it may aid in establishing a positive correlation with variety of occlusal traits or deleterious habits established early in life. It can also be performed in different ethnic populations to validate the association of ethnicity with shape of PR. Hence, future scope of present study on a larger sample size may support the use of PR not only for forensic identification but also as a diagnostic appurtenance of malocclusion.

\section{Acknowledgement}

Sincere thanks to my colleagues, Dr Panchali Batra and Dr Anurag Negi for inclusion of the casts of their patients for the present sample.

\section{References}

1. Patil MS, Patil SB, Acharya AB (2008) Palatine rugae and their significance in clinical dentistry: a review of the literature. J Am Dent Assoc 139: 1471-1478.

2. Sivaraj A (2013) Significance of ruga in orthodontics J Orofac Res 3(3): 202-209.

3. Lysell L (1955) Plicae palatinae transversae and papilla incisiva in man; a morphologic and genetic study. Acta Odontol Scand 13(18): 5-137.

4. Buchtová M, Tichy F, Putnová I, Mísek I (2003) The development of palatal rugae in the European pine vole, Microtus subterraneus (Arvicolidae, Rodentia) Folia Zoo 52: 127-136

5. Sadler TW (1990) Langman's medical Embriology: Williams and Wilkins, Baltimore, 316-320.

6. Carrea JU (1937) La Identificacionhumanaporlasrugosidadespalatinas Rev Orthodont (Buenos Aires) 1: 3-23

7. Gandikota C, Venkata YP, Challa P, Juvvadi SR, Mathur A (2012) Comparative study of palatal rugae pattern in class II div 1 and class I individuals. J Pharm Bioallied Sci 4: S358-363.

8. Jordanov JA (1971) [Growth of the hard palate in man. Anthropological characteristics]. Z Morphol Anthropol 63: 230-237.

9. Lang J, Baumeister R (1984) [Postnatal development of the width and height of the palate and the palate foramina]. Anat Anz 155: 151-167.

10. van der Linden FP (1978) Changes in the position of posterior teeth in relation to ruga points. Am J Orthod 74: 142-161.

11. Kapoor P, Miglani R (2015) Transverse changes in lateral and medial aspects of palatal rugae after mid palatal expansion: A pilot study. J Forensic Dent Sci 7(1): 8-13.

12. Almeida MA, Phillips C, Kula K, Tulloch C (1995) Stability of the palatal rugae as landmarks for analysis of dental casts. Angle Orthod 65: 43-48.

13. Bailey LT, Esmailnejad A, Almeida MA (1996) Stability of the palatal rugae as landmarks for analysis of dental casts in extraction and nonextraction cases. Angle Orthod 66: 73-78.

14. Jang I, Tanaka M, Koga Y, lijima S, Yozgatian JH, et al. (2009) A novel method for the assessment of three-dimensional tooth movement during orthodontic treatment. Angle Orthod 79: 447-453.

15. Ritter R (1943) Uber die Form, den Verlauf und die Typeneinteilung der Gaumenleisten. Zeitschrift fir Morphologie und Anthropologie 40: 367-372.

16. Nilles K (1950) Morphologishche und erbbiologische Studienuber Gaumenmuster. Inaugural dissertation, Heidelberg (Typescript).

17. Nilles K (1952) [The palate relief as a diagnostic help in the anthropologic proof of paternity]. Stoma (Heidelb) 5: 121-125.

18. Kapali S, Townsend G, Richards L, Parish T (1997) Palatal rugae patterns in Australian aborigines and Caucasians. Aust Dent J 42: 129-133.

19. Hauser G, Daponte A, Roberts MJ (1989) Palatal rugae. J Anat 165: 237-249.

20. Eisentraut (1976) Das Gaumenfaltenmuster der Säugetiere und seine Bedeutungfür Stammesgeschichtliche und taxonomische Untersuchungen Bonn.

21. Kallianpur S, Desai A, Kasetty S, Sudheendra U, Joshi P (2011) An anthropometric analysis of facial height, arch length, and palatal rugae in the Indian and Nepalese population. J Forensic Dent Sci 3: 33-37.

22. Lux CJ, Conradt C, Burden D, Komposch G (2003) Dental arch widths and mandibular-maxillary base widths in Class II malocclusions between early mixed and permanent dentitions. Angle Orthod 73: 674-685.

23. Uysal T, Usumez S, Memili B, Sari Z (2005) Dental and Alveolar Arch Widths in Normal Occlusion and Class III Malocclusion Angle Orthod 75: 809-813.

24. Kashima K (1990) [Comparative study of the palatal rugae and shape of the hard palatal in Japanese and Indian children]. Aichi Gakuin Daigaku Shigakkai Shi 28: 295-320.

25. Shetty SK, Kalia S, Patil K, Mahima VG (2005) Palatal rugae pattern in Mysorean and Tibetan populations. Indian J Dent Res 16: 51-55.

26. Laganà G, Masucci C, Fabi F, Bollero P, Cozza P (2013) Prevalence of malocclusions, oral habits and orthodontic treatment need in a 7 - to 15-yearold schoolchildren population in Tirana. Prog Orthod 14: 12.

27. Primozic J, Farcnik F, Perinetti G, Richmond S, Ovsenik M (2013) The association of tongue posture with the dentoalveolar maxillary and mandibular morphology in Class III malocclusion: a controlled study. Eur J Orthod 35: 388-393.

28. Yilmaz F, Sagdiç D, Karaçay S, Akin E, Bulakbasi N (2011). Tongue movements in patients with skeletal Class II malocclusion evaluated with real-time balanced turbo field echo cine magnetic resonance imaging Am J Orthod Dentofacial Orthop139(5): 415-425

29. Jena AK, Duggal R, Mathur VP, Parkash H (2005) Class-III malocclusion: genetics or environment? A twins study. J Indian Soc Pedod Prev Dent 23 27-30.

30. Cakan DG, Ulkur F, Taner TU (2012) The genetic basis of facial skeletal characteristics and its relation with orthodontics. Eur J Dent 6: 340-345.

31. Limson KS, Julian R (2004) Computerized recording of the palatal rugae pattern and an evaluation of its application in forensic identification. J Forensic Odontostomatol 22: 1-4.

32. Shetty M, Premlatha K (2011) Study of Palatal Rugae Pattern among the Student Population in Mangalore J Indian Acad Forensic Med. 33(2): 112-115.

33. Shukla D, Chowdhry A, Bablani D, Jain P, Thapar R (2011) Establishing the reliability of palatal rugae pattern in individual identification (following orthodontic treatment). J Forensic Odontostomatol 29: 20-29. 